\title{
Aportes a la distribución del género Micrurus (Serpentes: Elapidae) en el Departamento del Chocó, Colombia
}

\section{Contributions to the distribution of the genus Micrurus (Serpentes: Elapidae) in the Department of Chocó, Colombia}

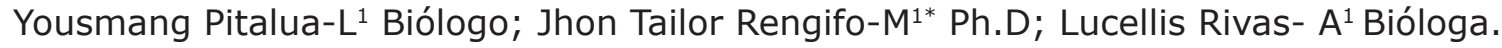

${ }^{1}$ Universidad Tecnológica del Chocó, "Diego Luis Córdoba" Ciudadela Universitaria B/ Nicolás de Medrano, bloque 11, oficina 412, Quibdó, Colombia.

\section{KEYWORDS:}

Micrurus;

snakes;

potential distribution;

Chocó;

Colombia.
PALABRAS CLAVE:

\section{Micrurus;}

serpientes;

distribución potencial;

Chocó;

Colombia.

\section{ABSTRACT}

We studied the potential distribution and richness of coral snakes, genus Micrurus (Serpentes: Elapidae), for the tropical rain forest in the department of Chocó; Through a detailed review of collections in the SIB-Colombia databases (Biodiversity Information System-Colombia) for the distribution of potential, the Maxen program was used, generating a map for each species. The review showed a total of 145 individuals in collections of the seven species reported for Chocó, deposited in eight scientific collections of the SIB; the species $M$. dumerilli and $M$. ancorali, were those that presented the most in the collections consulted; M. dumerilli is one of the most widely distributed species because it remains in the rainforests colonizing different microhabitats, followed by M. ancoralis that is distributed in the department of Chocó, Cauca, Risaralda, Nariño and Valle del Cauca; On the other hand, in the analysis of endemic species it was found that $M$. spurelli is a species that is only distributed in the department of Chocó. The subregions of the south and the center harbor the greatest wealth of this genus, thus obtaining $100 \%$ of the species that are reported for the department of Chocó.

\section{RESUMEN}

Se estudió la distribución potencial de las serpientes de coral, género Micrurus (Serpentes: Elapidae), para el bosque pluvial tropical en el departamento del Chocó; por medio de una revisión detallada de colecciones en la bases de datos del SIB-Colombia (Sistema de Información de Biodiversidad-Colombia) para la distribución potencial se utilizó el programa Maxen, generando un mapa para cada especie. La revisión arrojo un total de 145 individuos en colecciones de las siete especies reportadas para el Chocó, depositados en ocho colecciones científicas del SIB; las especies M. dumerilli y M. ancorali, fueron las que más registro presentaron en las colecciones consultadas; $M$. dumerilli es una de las especies de mayor distribución pues permanece en las selvas tropicales colonizando distintos microhábitats, seguida de M. ancoralis que se distribuye en el departamento del Chocó, Cauca, Risaralda, Nariño y Valle del Cauca; por otro lado, en el análisis de especies endémicas se encontró que $M$. spurelli es una especie que solo se distribuye en el departamento del Chocó. Las subregiones del sur y del centro albergan la mayor riqueza de este género, obteniendo así el $100 \%$ de las especies que son reportadas para el departamento del Chocó. 


\section{INTRODUCCIÓN}

En Colombia existen aproximadamente 270 especies de serpientes, agrupadas en las familias Aniliidae, Anomalepididae, Boidae, Colubridae, Elapidae, Hidrophydae, Leptotyphlopidae, Tropidophidae, Typhlopidae, y Viperidae, las cuales están distribuidas en casi todo el país, exceptuando las aguas del Caribe, y las tierras por encima de los 3.500 msnm (LLANO-MEJIA et al., 2010; LYNCH, 2012). Estas se agrupan en 27 familias con alrededor de 3.450 especies en el mundo (VIDAL y HEDGES, 2002; ZAHER et al., 2009; GRAZZIOTIN et al., 2012; PINCHEIRA-DONOSO et al., 2013; PYRON et al., 2013; UETZ y HALLERMANN, 2014). Este grupo se puede encontrar desde los ecosistemas secos o desérticos, hasta los bosques húmedos e hiperpluviales del mundo, donde la diversidad más alta del grupo se encuentra en las regiones tropicales; la familia Elapidae tiene alrededor de 359 especies, distribuidas desde el sureste y suroeste de los Estados Unidos, a través de México, América Central y América del Sur, también se encuentran en Asia, África y Australia; en América, hay un grupo de más de 120 especies y subespecies, dividido en cuatro géneros: Micruroides, Leptomicrurus, Pelamis y Micrurus (UETZ et al., 2016).

Los Elápidos del género Micrurus son una de las familias más diversas de Colombia, lo que les ha permitido encontrarse en distintos hábitats como en zonas boscosas, semiboscosa, parches de bosques, bosques húmedos pluvial tropical, pre montano y en ocasiones cerca de asentamientos humanos, donde hay presencia de minería y agricultura. En Colombia, el género Micrurus está representado por 24 especies aproximadamente; las serpientes de este género presentan hábitos fosoriales, semifosoriales, crepusculares y una dentición proteroglifa, produciendo un potente veneno de efecto neurotóxico y son conocidas comúnmente como serpientes de coral (BOLAÑOS,1982); una de las regiones naturales de Colombia que alberga una diversidad biológica importante, es la región del Chocó, donde se registran siete especies de Micrurus (M. ancoralis, M. spurelli,
M. mipartitus, M. dumerilli, M. clarki, M. dissoleucus, M. multicustatus), distribuidas en todo el territorio por debajo de los 2.000 $m$ de altitud (RENFIGO y RENTERIA, 2011).

Las causas de la rápida declinación de las poblaciones de serpientes de coral a nivel nacional, involucran diversos factores que actúan de manera sinérgica y reducen aún más las posibilidades de conservación de los taxones amenazados; una de las mayores amenazas para la biodiversidad no sólo de serpientes de coral, sino de otros grupos animales es la destrucción y el deterioro de hábitats naturales, en Colombia y en el Chocó esto no es una excepción, el Instituto de Hidrología, Meteorología y estudios Ambientales (IDEAM), registro un 16,30\% de transformación de los bosques entre 1986-1996 (RUEDA y MARTÍNEZ, 2004). La pérdida de diversidad biológica, producto del deterioro del hábitat, está afectando un alto porcentaje de las serpientes de coral, las cuales son una importante porción de la biomasa de vertebrados y están desapareciendo a tasas elevadamente altas, incluso en ambientes prístinos, las poblaciones de serpientes de coral han ido desapareciendo, el $40 \%$ de las especies se encuentran amenazadas (YOUNG et al., 2004).

La destrucción de los hábitats según YOUNG et al. (2001), STUART et al. (2004) y LIPS et al. (2003) está ocupando el primer lugar entre las causas identificadas por las cuales se está perdiendo riqueza de especies a nivel global, otros investigadores expresan causas como los cambios climáticos (POUNDS y CRUMP, 1994; POUNDS et al., 1999; POUNDS y PUSCHENDORF, 2004), la introducción de nuevas especies exóticas (KUPFERBERG, 1997; KIESECKER et al., 2001a), el empleo masivo de pesticidas, agroquímicos en la agricultura y las fumigaciones con glifosato (RELYEA, 2005); sumado a esto la matanza por la Ofidiofobia, la mortalidad vehicular de serpientes de coral sobre carreteras, la eliminación de las presas de las serpientes y la recolección científica, son otros factores que están ejerciendo una fuerte presión sobre la densidad de estas serpientes (LYNCH, 2012). 
Pitalua-L et al - Aportes a la distribución del género Micrurus (Serpentes: Elapidae) Chocó, Colombia.

PEE⿱

A pesar de tanta diversidad y riqueza de especie de serpientes de corales, su actividad se ha visto amenazada innumerablemente por factores que reducen la densidad y diversidad de sus especies, por lo tanto, en esta investigación se estudió la distribución potencial y riqueza de las serpientes de coral, género Micrurus (Serpientes: Elapidae), reportadas para el bosque pluvial tropical del departamento del Chocó, Colombia.

\section{MATERIALES Y MÉTODOS}

Área de estudio. Las zonas objeto de estudio, se localizan en las llanuras aluviales y colinas bajas del valle del río Atrato, en municipios aledaños a los alrededores de Quibdó, capital del departamento del Chocó, donde se concentra la mayor pluviosidad del andén del Pacífico y donde las formaciones selváticas se encuentran entre las más ricas del mundo (CUATRECASAS, 1958; FORERO y GENTRY, 1989). Los puntos de muestreos están incluidos en las coordenadas geográficas $5^{\circ} 00^{\prime}-6^{\circ} 45^{\prime} \mathrm{N}$ y 77015'-76030' W denominado selva pluvial central. La temperatura está entre megatermal bajo $\left(23,5\right.$ a $\left.25,7^{\circ} \mathrm{C}\right)$ y megatermal $(25,7$ a $\left.27,9^{\circ} \mathrm{C}\right)$, con promedio anual de $23,5^{\circ} \mathrm{C}$ (POVEDA et al., 2004). En el valle del río Atrato, se ubicaron cinco municipios de la selva pluvial central (Certegui, Unión Panamericana (Salero), Lloro, Atrato (Samurindó) y Quibdó (Pacurita).

Levantamiento de Información Secundaria. Se realizó una revisión detallada de las bases de datos de fauna de la Universidad Tecnológica del Chocó (COLZOOCH-H), Universidad de la Sallé, Alexander von Humboldt, Universidad del Valle, Corporación Autónoma Regional del Valle Cauca (CVC), Universidad Pontificia Javeriana, Corporación Autónoma Regional del Centro de Antioquia (CORANTIOQUIA) y Universidad de Antioquia, ubicadas en el portal de datos del SIB-Colombia.

Determinación Taxonómica. Esta se realizó con la ayuda de claves y guías prácticas de PETERS y OREJAS (1970), PÉREZ y MORENO (1988), PÁEZ y BRIAN (2002) y comparaciones con material de la Colección Científica de Referencia de la Fauna chocoana, con sede en la Universidad Tecnológica del Chocó; para establecer la distribución potencial de las especies de serpientes de coral se utilizó, el programa de modelamiento MaxEnt; la idea básica de MaxEnt es estimar (aproximar) la probabilidad de distribución desconocida de una especie (PHILLIPS et al., 2006), por lo tanto los datos fueron suministrados para que Maxent acoplarálos archivos de Excel con las siguientes características: Nombre de especies y sus respectivas coordenadas geográficas Ejemplo: Micrurus mipartitus 4.15, -74.25, para después guardar este archivo con extensión ".csv".

Una vez ejecutado el programa se descartó las variables ambientales que mediante el análisis jackknife aportan menos del $10 \%$ al modelo, después de descartar estas variables se corrió nuevamente el programa para hallar la distribución potencial para cada especie; uno de los parámetros que utiliza el Maxent para evaluar la capacidad de predecir sus modelos es el área por debajo de la Curva o estadístico (AUC); la interpretación del AUC para los modelos generados es la siguiente: Excelente si el AUC>0.90; Buena si 0.80>AUC<0.90; Aceptable si $0.70>$ AUC $<0.80$; Mala si $0.60>$ AUC $<0.70$; No válida si $0.50>A U C<$ 0.60 .

Las imágenes o mapas arrojados por el Maxent, usan los colores para indicar la probabilidad de que las condiciones sean adecuadas, en el caso de estudio, el verde oscuro, indica una alta probabilidad de condiciones adecuadas para la especie, el rojo indica las condiciones típicas de aquéllos lugares donde la especie se encuentra y el verde opaco indica una baja probabilidad de condiciones adecuadas para que las especies ocurran.

\section{RESULTADOS Y DISCUSIÓN}

Con un esfuerzo de muestreo de $480 \mathrm{~h}$.h se registraron tres individuos, para un éxito de captura de 0,006ind/h.h, el escaso éxito de captura en este estudio pudo estar influenciado por la temporada de Iluvia, 
siendo estos meses de poco volumen de Iluvia; según LYNCH (2012) estas serpientes se ven con poca frecuencia sobre la superficie del suelo, dado a sus hábitos minadores o fosoriales, sumado a esto, las serpientes son uno de los grupos que presentan la mayor cantidad de amenazas, como la matanza por ofidiofobia, la matanza de serpientes por vehículos en la carretera, la destrucción o intervención de los habitas naturales, el tráfico ilegal y la recolección científica.

Para Colombia según LYNCH (2012) y CASTAÑO et al. (2004), el género Micrurus ocupa el tercer lugar en riqueza especies con aproximadamente 22spp., mientras que para el Chocó Biogeográfico, ocupa el segundo con nueve especies distribuidas por su territorio por debajo de los $2000 \mathrm{~m}$ de altitud, en lo que radica la importancia de este grupo, ya que en Colombia solo son superadas por los géneros Anolis y Atractus (CASTAÑO et al., 2004).

La revisión documental realizada a la base de datos del SIB Colombia, reportó 145 individuos, (RENGIFO y RENTERÍA, 2011), de siete especies para el departamento del Chocó (Tabla 1), 37 se registran para la región natural chocoana; para un mejor análisis sobre la distribución de las especies se dividió el departamento del Chocó en cinco subregiones (Región Pacífica, Región Norte, Región Sur, Región Central y Región Montañosa), encontrando que la subregión del Sur presenta la mayor riqueza de serpientes de coral, albergando así las siete especies descritas para el Departamento.
En términos de exclusividad esta región presentó una especie $M$. dissoleucus, según, los estudios realizados por CAMPBELL y LAMAR (2004), esta especie ocurre en la región norte del Chocó, y en el valle del Magdalena; esta especie también es reportada para la región del Atlántico y para el norte de Colombia (CASTAÑO et al., 2004), quienes realizaron la revisión de los reptiles para la región del Chocó biogeográfico, indican la ocurrencia de esta especie, en las tierras bajas del departamento del Chocó, encontrado un registro en el Municipio de Andagoya, Chocó. ROZE (1996) explica que estas especies prefieren los bosques húmedos tropicales y bosques húmedos montanos bajos, sin embargo CAMPEBELL y LAMAR (2004) aseguran que especies fosoriales y semifosorial, rara vez se ven y seguirán siendo desconocidas en gran parte de su rango de alcance y tamaño de poblaciones si no se llevan a cabo encuentros específicos.

La segunda zona de importancia en riqueza de corales es la subregión del centro del departamento, conocida como Atrato, cabe destacar que para esta subregión, PALACIOS y RENGIFO (2012) registraron una nueva localidad de distribución para M. Spurelli, la cual presentaba una restringida distribución en la región chocoana, además presentan el primer registro de $M$. multiscutatus para el departamento del Chocó. Con base a los registros realizados a la base de datos del SIB Colombia, se estimó que las especies más representativas, por su abundancia son, M. dumerilli con $31,5 \%$ y $M$. ancoralis con $25,7 \%$, debido aquel resto de las especies en

Tabla 1. Revisión del genero Micrurus en el departamento del Chocó.

\begin{tabular}{|c|c|c|c|c|c|c|}
\hline \multirow[b]{2}{*}{ Especie } & \multicolumn{5}{|c|}{ Regiones } & \multirow[t]{2}{*}{ Registro } \\
\hline & $\mathbf{R N}$ & $\mathbf{R P}$ & RC & RS & $\mathbf{R M}$ & \\
\hline M. ancoralis (Jan, 1898) & 1 & 0 & 1 & 1 & 1 & $1,2,3,4,6,7$ \\
\hline M. dumerilli (Schimidt, 1936) & 1 & 1 & 1 & 1 & 1 & $1,2,3,4,6,7$ \\
\hline M. spurelli (Cope, 1936) & 0 & 0 & 1 & 1 & 0 & 4,8 \\
\hline M. multiscutatus (Griffin, 1916) & 0 & 0 & 1 & 1 & 0 & 4,8 \\
\hline M. mipartitus, (Dumeril, 1854) & 0 & 1 & 0 & 1 & 1 & $2,4,7$ \\
\hline M. dissoleucus (Rendahl y Vestergren, 1940) & 0 & 0 & 0 & 1 & 0 & 4,7 \\
\hline M. clarki (Girard, 1855) & 0 & 0 & 1 & 1 & 0 & 4,7 \\
\hline
\end{tabular}

1, MOSQUERA (2015), 2. ECHAVARRÍA (2014), 3. RENTERÍA (2011), 4. RENGIFO (2006), 5. MORENO (2006), 6. MURILLO (2004), 7. CASTAÑO (2004), 8. PALACIOS (2012). 
Pitalua-L et al - Aportes a la distribución del género Micrurus (Serpentes: Elapidae) Chocó, Colombia.

común reportan el $42,8 \%$ de los individuos que presentaron abundancia relativamente baja. La gran abundancia de estas dos especies puede estar asociada, con su fácil adaptación y cambios de microhábitats, ya que estas especies ocurren en bosques húmedos, pluvial tropical, premontanos, bosques secos, en zonas intervenidas, Io que les ha permitido ampliar su rango de distribución y albergar una gran variedad de ambientes, como se evidencia en los estudios realizados por CASTAÑO (2004), MURILLO et al. (2006), RENTERÍA et al. (2011) y MOSQUERA (2016), en los cuales estas especies son las más representativas del género Micrurus, para el departamento del Chocó.

Según el estado de conservación de las siete especies registradas para el departamento del Chocó solo M. clarki y M. dissoleucus, se encuentran en preocupación menor (BATISTA et al., 2014; IBAÑEZ et al., 2015), datos publicados en la lista roja de la UICN (2015). En términos de endemismos $M$. spurelli, especie endémica del departamento del Chocó, se encuentra en categoría de casi amenazada, debido a su restringida distribución y a los diferentes procesos antrópicos a los que está sometido el hábitat que ocupa esta especie BATISTA et al. (2014), de igual manera ARTEAGA et al. (2017) expresan que M. multiscutatus, especie casi endémica del Chocó biogeográfico colombiano, ya que su distribución se extiende hasta el Ecuador, se encuentra en categoría de casi amenazada, con alta probabilidad de pasar a vulnerable, ya que ha venido disminuyendo su distribución y registros, lo que puede ser atribuido a la perdida de hábitat, ya que la mayoría de los bosques de tierras bajas en la distribución de esta especie han sido destruidos a causa de la minería, deforestación, agricultura y cultivos ilícitos, como lo señalan BATISTA et al., 2014, en la lista roja de la UICN. Para la distribución potencial se tuvo en cuenta los 145 individuos registrados y se obtuvo que el $60 \%$ de los modelos tuvieron un desempeño (Excelente), y el 40\% (Bueno) (Tabla 2).
Tabla 2. Resultado del estadístico AUC, para las especies del genero Micrurus.

\begin{tabular}{lcccc}
\hline \multicolumn{1}{c}{ Especies } & N reg. & AUC & Clase & Área $\mathbf{~ k m}^{2}$ \\
\hline M. ancoralis & 22 & 0.96 & $\mathrm{E}$ & 24.368 \\
M. dumerilli & 46 & 0.93 & $\mathrm{E}$ & 201.303 \\
M. Spurelli & 3 & 0.86 & $\mathrm{~B}$ & 250 \\
M. multiscustatus & 7 & 0.91 & $\mathrm{E}$ & 12.500 \\
M. mipartitus & 46 & 0.95 & $\mathrm{E}$ & 106.76 \\
M. dissoleucus & 15 & 0.85 & $\mathrm{~B}$ & 82.683 \\
M. clarki & 6 & 0.86 & $\mathrm{E}$ & 188.102 \\
\hline \multicolumn{1}{c}{ Total } & $\mathbf{1 4 5}$ & & & \\
\hline
\end{tabular}

$(\mathrm{E}=$ Excelente, $\mathrm{B}=$ Bueno $)$

M. ancoralis: El área potencial que esta especie ocupa es de $24.368 \mathrm{~km}^{2}$ aproximadamente, la cual se distribuye desde 534 '57", 76³8'58" (Samurindó), hasta $3^{\circ} 53^{\prime} 25^{\prime \prime}, 77^{\circ} 4^{\prime} 43^{\prime \prime}($ Buenaventura), (Figura 1), según RENGIFO et al. (2004) y los resultados de la distribución potencial indican que para los bosques húmedos, pluvial y premontanos, la especie $M$. ancoralis, encuentra las condiciones de los hábitat para su desarrollo ocurriendo con mayor presencia en los departamentos del Chocó, Cauca, Risaralda, Nariño y Valle del Cauca, por debajo de los 2.000 msnm, con media y baja probabilidad en bosques nublados.

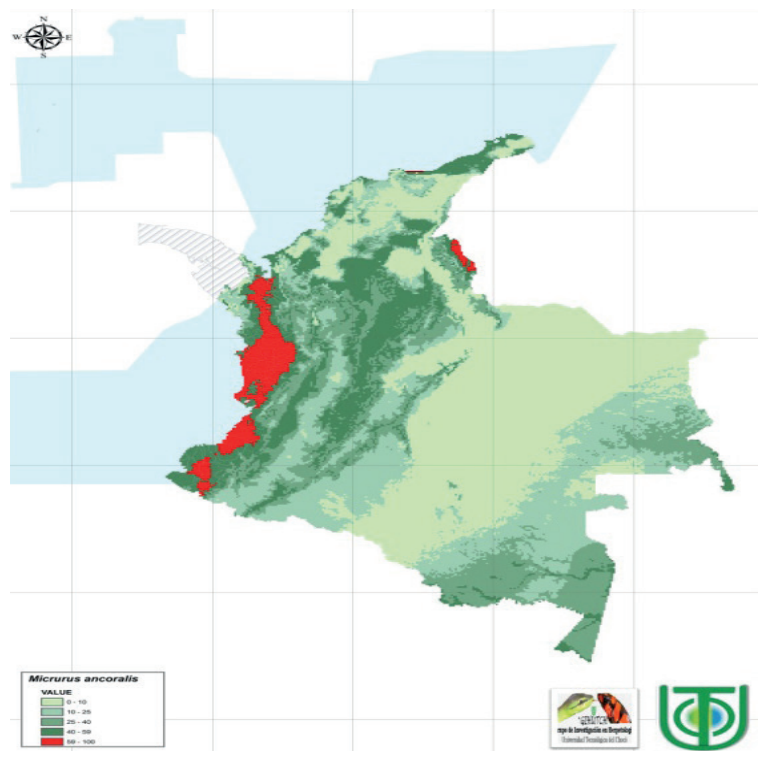

Figura1. Distribución potencial de $M$. ancoralis en el departamento del Chocó, Colombia. 
RENGIFO y RENTERÍA (2011) reportan la especie al oeste de los Andes en la llanura del Pacifico y en las estribaciones de la cordillera Occidental, por debajo de los $1.000 \mathrm{msnm}$, autores como MOSQUERA (2016), ECHAVARRÍA y DELGADO (2009), RENGIFO et al. (2002), MURILLO (2006) y CASTAÑO et al. (2004) la registran en los municipios de Quibdó (Pacurira), Atrato (Samurindo), Istmina, Andagoya, Lloro y Unión Panamericana (Salero). Las variables con mayor contribución al modelo es: la pre6190_ann o precipitación anual con $(61 \%)$, seguida de precipitación 6190_110 o precipitación de octubre con el (38\%).

M. dumerilli: El área potencial que esta especie ocupa es de $201.303 \mathrm{~km}^{2}$ aproximadamente, la cual se distribuye

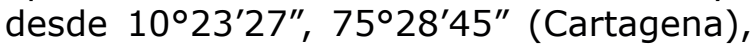
hasta 1017'20", 77021'28", (Nariño), (Figura 2), según, RENGIFO y RENTERÍA (2011), y los resultados de la distribución potencial, $M$. dumerilli, se encuentra con alta probabilidad de ocurrencia en bosques húmedos, bosque de niebla, selva tropical seca, selva tropical lluviosa, y matorral espinoso, encontrándose en los departamentos del Chocó, Valle del Cauca, Tolima, Cundinamarca, Santander, Antioquia, Caldas, Bolívar, Córdoba y Nariño, ocurriendo por debajo de los 1.750 msnm.

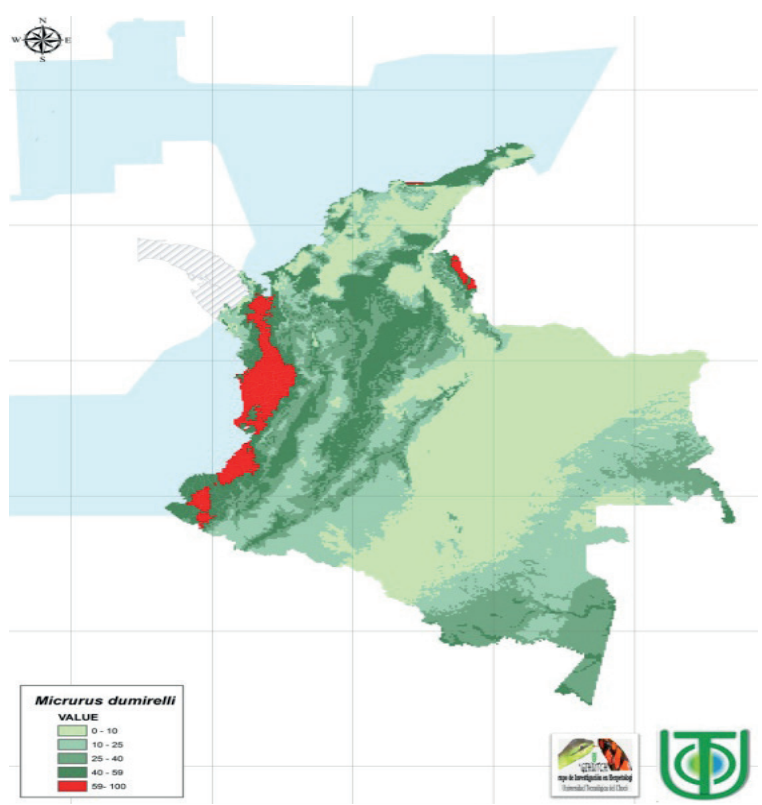

Figura 2. Distribución potencial de M. dumirelli en el departamento del Chocó, Colombia.
Según MURILLO (2006), esta especie se ha registrado en la región Andina y en las cordilleras Occidental y Central por debajo de los $1.700 \mathrm{msnm}$; autores como, MOSQUERA (2016), ECHAVARRÍA (2009), RENGIFO et al. (2004) la reportan en los municipios de Novita, Lloro, Atrato (San Martin y San José de Purre), Quibdó (Cabecera Municipal, Pacurita, Samurindo, Tutunendo, Guadalupe) y en Unión Panamericana (Salero). Las variables con mayor contribución al modelo es: la pre6190_110 o precipitación de octubre con (62\%), seguida de pre6190_ ann o precipitación anual con el $(37 \%)$.

M. clarki: El área potencial que esta especie ocupa es de $188.102 \mathrm{~km} 2$ aproximadamente, la cual se distribuye desde $7^{\circ} 57^{\prime} 40^{\prime \prime}, 72^{\circ} 41^{\prime} 0^{\prime \prime}$, (Norte de Santander), hasta 77021'28', (Nariño) (Figura 3). SAVAGE (2002) y CAMPBELL y LAMAR (2004), los resultados de la distribución potencial, M. clarki, se encuentra con alta probabilidad de ocurrencia en selvas tropicales lluviosas bosques húmedos de tierras bajas, selva tropical premontano, zonas más secas como áreas de transición entre selva tropical húmeda y seca, en esta última asociada a los hábitats ripiaros, lo que concuerda con JARAMILLO et al. (2012), quien las reporta en los departamentos del Chocó, Valle del Cauca, Cauca, Nariño, Santander y Cundinamarca, ocurriendo por debajo de los 700 msnm; RENGIFO y RENTERÍA (2011) y CASTAÑO et al. (2004) esta especie ocurre en el departamento del Chocó en los municipio de Istmina (Andagoya) y Rio Sucio.

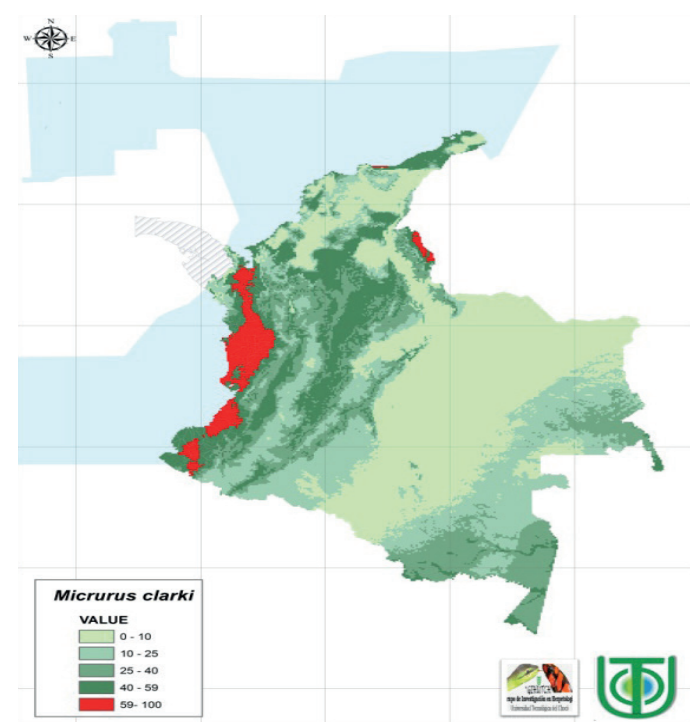

Figura 3. Distribución potencial de M. clarki en el departamento del Chocó, Colombia. 
M. dissoleucus: El área potencial que esta especie ocupa es de $82.683 \mathrm{~km}^{2}$ aproximadamente, la cual se distribuye desde $11^{\circ} 26^{\prime} 28^{\prime \prime}, 72^{\circ} 32^{\prime} 54^{\prime \prime}$, (Guajira), hasta $5^{\circ} 5^{\prime} 43^{\prime \prime}, 76^{\circ} 41^{\prime} 43^{\prime \prime}$, (Andagoya) (Figura 4). Según LAVIN y DIXON (2004), los resultados de la distribución potencial, M. dissoleucus, se encuentra con alta probabilidad de ocurrencia en selva seca tropical, bosque montañoso bajos, matorral espinoso, matorral costero, llanos y sabanas, siendo registrada con mayor frecuencia en zonas secas séricas, con poca ocurrencia en bosques húmedos, encontrándose en los departamentos de Cúcuta, Chocó, Magdalena, Bolívar, Sucre, Guajira, Santander, Santa Marta y Barranquilla por debajo de los 1.000 msnm como lo reportan CAMPBELL y LAMAR (2004), RENGIFO et al. (2004) reportan esta especie para las tierras bajas del departamento del Chocó en el municipio de Istmina (Andagoya).

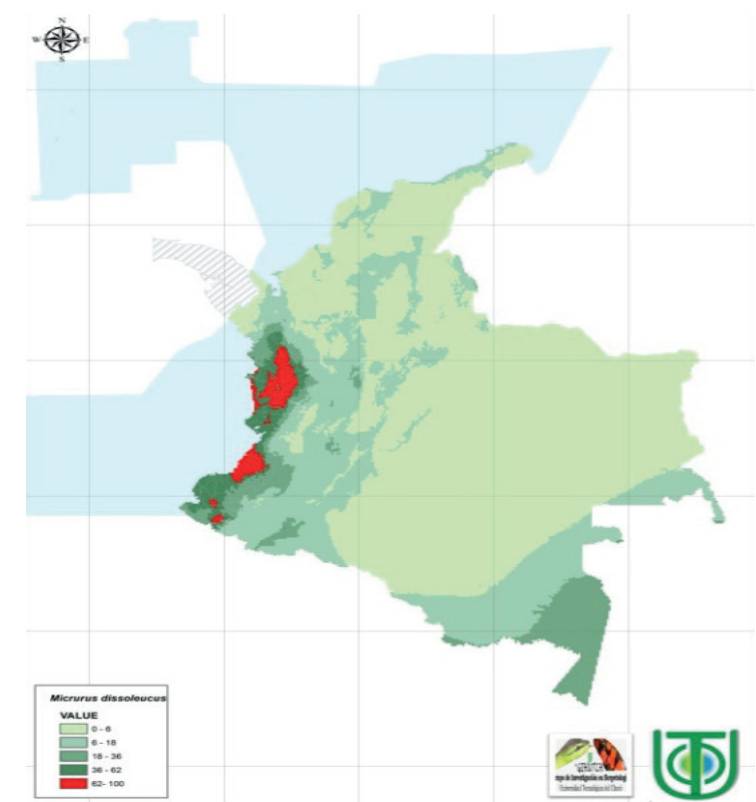

Figura 4. Distribución potencial de $M$. dissoleucos en el departamento del Chocó, Colombia.

M. mipartitus: El área potencial que esta especie ocupa es de $106.76 \mathrm{~km}^{2}$ aproximadamente, la cual se distribuye desde $10^{\circ} 22^{\prime} 39^{\prime \prime}, 74^{\circ} 15^{\prime} 41^{\prime \prime},($ Magdalena), hasta $2^{\circ} 47^{\prime} 50^{\prime \prime}, 76^{\circ} 38^{\prime} 26^{\prime \prime}$, (Cauca) (Figura $5)$, según los resultados de la distribución potencial, M. mipartitus, se encuentra con alta probabilidad de ocurrencia en bosques bajos montañosos, bosques de niebla y matorrales secos y rocosos, encontrándose en los departamentos de Cauca, Valle del Cauca, Meta, Chocó, Cundinamarca, Boyacá, Caldas, Magdalena, Antioquia, Huila, Bolívar, Santander y Risaralda, por debajo de los $2.410 \mathrm{msnm}$.

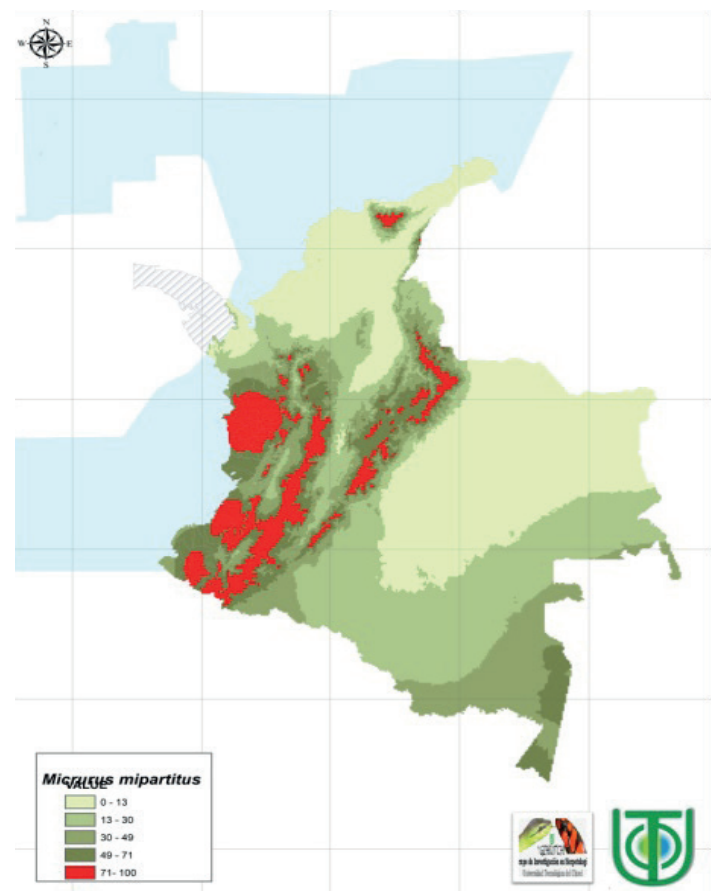

Figura 5. Distribución potencial de M. mipartitus en el departamento del Chocó, Colombia.

M. multiscutatus: El área potencial que esta especie ocupa es de $12.500 \mathrm{~km}^{2}$ aproximadamente, la cual se distribuye desde 5०38'0", 76³1'59", (San José de Purre), hasta $2^{\circ} 27^{\prime} 9^{\prime \prime}, 76^{\circ} 48^{\prime} 40^{\prime \prime}$, (Tambo Cauca) (Figura 6). Según RENGIFO (2011) los resultados de la distribución potencial, M. multiscutatus, se encuentra con alta probabilidad de ocurrencia en bosques muy húmedos pluvial tropical, bosque tramoso, bosque húmedos premontanos, encontrándose en los departamentos de Cauca, Chocó y Valle del Cauca, por debajo de los 900 msnm, datos que concuerdan con ROZE (1996) y CAMPBELL y LAMAR (2004), reportan esta especie en el valle del rio Atrato. 


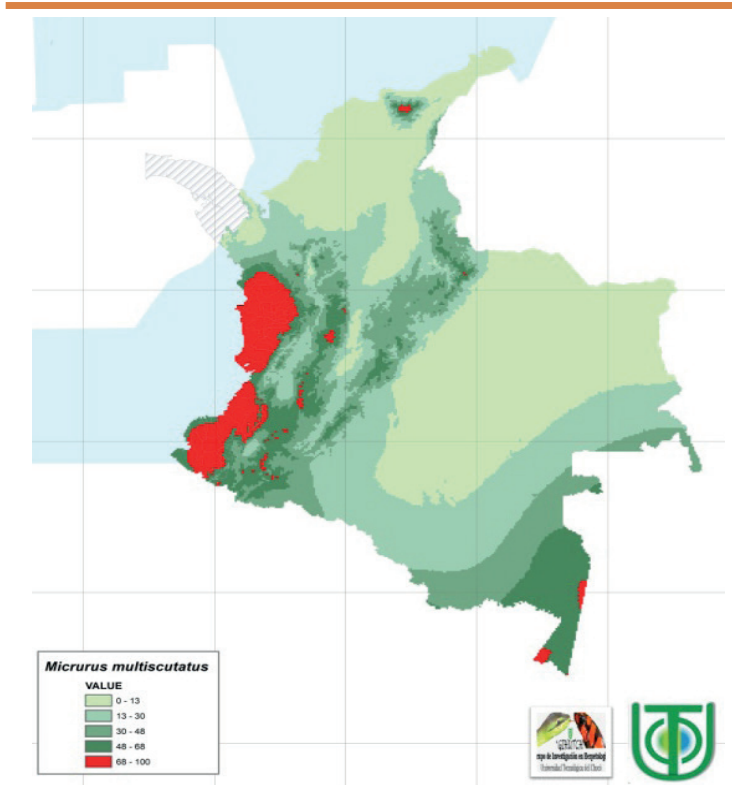

Figura 6.Distribución potencial de $M$. multicustatus en el departamento del Chocó, Colombia.

Cabe destacar que la distribución de $M$. multiscutatus, se ha venido disminuyendo alarmantemente encontrándose en un estado de casi amenazado y a punto de llegar a la categoría de vulnerable, esto debido a los procesos antrópicos que se vienen realizando en su hábitat, principalmente a la siembra de cultivos ilegales en bosques bajos como lo expresan ARTEAGA et al. (2017) en la UICN (2015).

M. spurelli: El área potencial que esta especie ocupa es de $250 \mathrm{~km}^{2}$ aproximadamente, la cual se distribuye desde 6 $6^{\circ} 40^{\prime} 27^{\prime \prime}$,

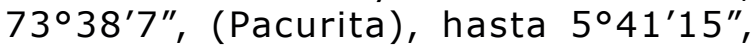
76०35'39", (valle del rio Atrato, (Figura 7). Según, PALACIOS y RENGIFO (2012) y los resultados de la distribución potencial, $M$. spurelli, se encuentra con alta probabilidad de ocurrencia en bosques húmedos, muy húmedos y pluvial tropical, encontrándose en el departamento del Chocó por debajo de los 250 msnm datos que concuerdan con CASTAÑO et al. (2004) quienes reportan la ocurrencia de esta especie en los municipios de Istmina y Condoto, de igual manera, RENGIFO y PALACIOS (2012) la reportan para el Valle del rio Atrato, en zonas intervenidas.

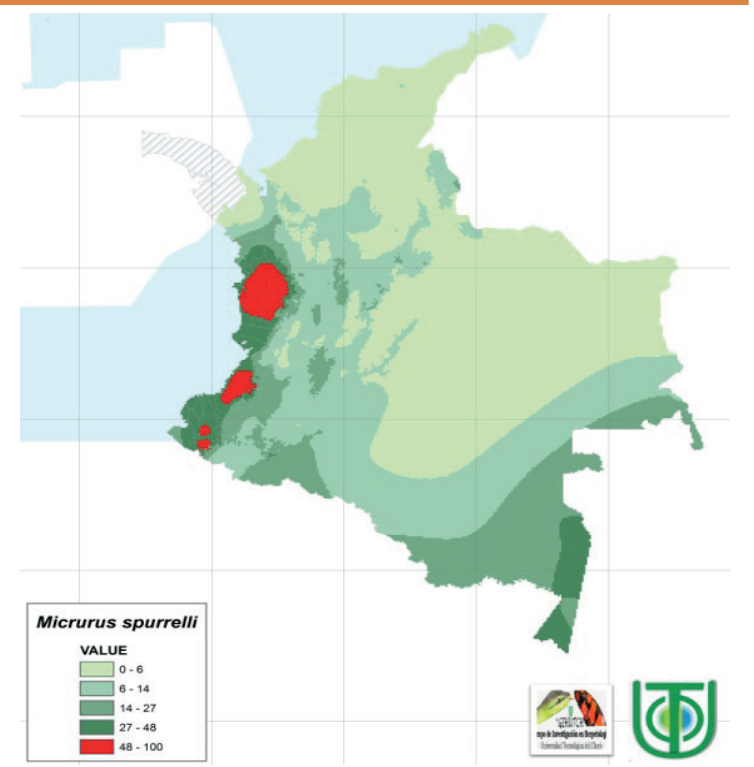

Figura 7. Distribución potencial de $M$. spurelli en el departamento del Chocó, Colombia.

En la Tabla 3 se presentan las variables de importancia obtenidas para el género Micrurus.

Tabla 3. Variables de importancia para el género Micrurus.

\begin{tabular}{lcc}
\hline Especie & Variable & $\begin{array}{c}\text { Porcent/ } \\
\text { Contribution }\end{array}$ \\
Micrurus ancoralis & pre6190_ann & 61 \\
& pre6190_110 & 38 \\
M. dumerilli & pre6190_110 & 57 \\
& pre6190_I1 & 28 \\
& h_dem & 28 \\
M. clarki & pre6190_110 & 27 \\
& frs6190_ann & 19 \\
& cld6190_ann & 12 \\
M. dissoleucus & tmx6190_ann & 11 \\
& pre6190_110 & 57 \\
M. mipartitus & pre6190_I1 & 28 \\
& tmn6190_ann & 13 \\
& pre6190_110 & 56 \\
M. multiscutatus & tmx6190_ann & 43 \\
& pre6190_110 & 45 \\
& cld6190_ann & 34 \\
& tmx6190_ann & 20 \\
M. spurelli & pre6190_110 & 37 \\
& tmx6190_ann & 34 \\
& frs6190_ann & 28 \\
\hline
\end{tabular}


Pitalua-L et al - Aportes a la distribución del género Micrurus (Serpentes: Elapidae) Chocó, Colombia.

\section{CONCLUSIONES}

Los modelos de la distribución potencial muestran que en algunas zonas las especies podrían afrontar problemas de conservación en el hábitat, que puede afectar la categoría de amenaza llevándola a la extinción local.

Según los reportes de especies del género Micrurus, para el departamento del Chocó la subregión del sur presenta las mejores condiciones para la ocurrencia de un mayor número de especies.
Los factores cambio climático y la ofidiofobia pese a no ser evaluados en este trabajo, algunos autores los catalogan como determinantes en la distribución potencial de las especies.

\section{Agradecimientos}

Expresamos nuestros agradecimientos al Grupo de Investigación en Herpetología de la Universidad Tecnológica del Chocó quienes nos proporcionaron los materiales y su colaboraron en la identificación y captura de las especies.

\section{REFERENCIAS}

ARTEAGA, A.; MEBERT, K.; VALENCIA, J.H.; CISNEROS-HEREDIA, D.F.; PEÑAFIEL, N.; REYESPUIG, C.; VIEIRA-FERNANDES, J.L.; GUAYASAMIN, J.M. 2017 molecular phylogeny of Atractus (Serpentes, Dipsadidae), with emphasis on Ecuadorian species and the description of three new taxa. Zookeys 661: 91-123.

BATISTA, A.; GARCÍA RODRÍGUEZ, A.; SABORÍO, G.; VARGAS ALVAREZ, J.; ACOSTA CHAVES, V.; RIVAS, G.; GUTIÉRREZ-CÁRDENAS, P.; CAICEDO, J.; RENJIFO, J. 2014. Micrurus clarki. The IUCN red list of threatened species 2014: e.t203624a2769200.

BOLAÑOS, R. 1982. Toxicity of Costa Rica snake venoms for the white mouse. amer. j. trop. med \& hyg.1972. 21: 360-363.

BOLAÑOS, R. 1982. Las serpientes venenosas de Centroamérica y el problema del ofidismo. Primera parte. Aspectos zoológicos, epidemiológicos y biomédicos. Rev. Cost. Ciencias Méd. 3: 165-184.

CAMPBELL, J.A.; LAMAR, W.W. 2004. The Venemous reptiles of the western hemisphere. Vol. (1) y ii. Cornell University, Ithaca. New York, Estados Unidos.

CASTAÑO-MORA, O.; CÁRDENAS-A. G.; HERNÁNDEZ-R.E.; CASTRO-H.F. 2004. Reptiles en el Chocó Biogeográfico. Págs. 599-632. En: RANGEL-CH. J.O. (Ed.). Diversidad Biótica IV. Universidad Nacional de Colombia, Instituto de Ciencias Naturales. Bogotá, Colombia.

COOK, F.R. 1965. Collecting and preserving amphibians and reptiles. Págs. 128-151. En; Anderson, R. M. (ed,.). Methods of collecting and preserving vertebrate animals. nat. mus. Canada, bull. 69, Biolo. ser. no. 18.

CUATRECASAS, J. 1958. Aspectos de la vegetación natural de Colombia. Revista de la Academia Colombiana de ciencias 10: 221-268.

CRUMP, M.; SCOTT, N. 1994. Visual encounter surveys, En Heyer, R.M.; Donnelly, Y.R.; Diarmid, A. ; Hayek, A.; Foster, M. (eds.). Measuring and monitoring biological diversity: standard methods for amphibians. Smithsonian Institution Press. Washington.

DOWLING, H.G.; DUELLMAN, W.E.1978. Systematic herpetology: A synopsis of families and higher categories. Hiss publications, New York, USA. 
ECHAVARRÍA-RENTERÍA, J.D.; DELGADO FRANCO, J.I. 2009. Caracterización espacio temporal de la comunidad de serpientes presentes en el corregimiento de Samurindó municipio del Atrato Chocó Colombia programa de biología, UTCH. Trabajo de grado. Biología.

FORERO, E.; GENTRY, A.H. 1989. Lista anotada de las plantas del departamento del Chocó, Colombia. Instituto de Ciencias Naturales, Museo de Historia Natural, Universidad Nacional de Colombia. Bogotá, Colombia.

GARCÍA, J.; CÁRDENAS, H.; CASTRO, F. 2007. Relación entre la diversidad de anuros y los estados sucesionales de un bosque muy húmedo montano bajo del Valle del Cauca, suroccidente colombiano. Caldasia 29 (2): 363-374

GRAZZIOTIN, F.G.; ZAHER, H.; MURPHY, R.W.; SCROCCHI, G.; BENAVIDES, M.A.; ZHANGY, Y.; BONATTOH, S.L. 2012. Molecular phylogeny of the New World Dipsadidae (Serpentes: Colubroidea): a reappraisal. Cladistics 1:1-23

GIBBONS, J.W.; SEMLITSCH, R.D. 1981. Terrestrial drift fences with pitfall traps: an effective technique for quantitative sampling of animal populations. Brimleyana 7: 1-16.

IBAÑEZ, R.; JARAMILLO, C.; STAFFORD, P.; MCCRANIE, J.R.; RENJIFO, J.; GUTIERREZCARDENAS, P.; CICEDO, J.; RIVAS, G. 2015. Micrurus dissoleucus. The IUCN red list of threatened species 2015: e t177541a1492349. International Union for Conservation of Nature and Natural Resources 2015

JARAMILLO, C.L.; WILSON, L.D.; IBAÑEZ, R.; JARAMILLO, F. 2010. The herpetofauna of Panama: distribution and conservation status. Págs. 604-671. En: Wilson, L.D.; Townsend, J.H.; Johnson, J.D. (eds.). Conservation of Mesoamerican Amphibians and Reptiles. Eagle Mountain Publishing, Eagle Mountain. Utah, USA.

JONES, B.K. 1981. Effects of grazing on lizard abundance and diversity in western Arizon. The southwestern naturalist 26: 105-107.

KIESECKER, J.M., BLAUSTEIN, A.R.; BELDEN, L.K. 2001a. complex causes of amphibian declines. Nature 410: 681-684.

KUPFERBERG, S.J. 1997. Bullfrog (Rana catesbeiana) invasion of a California river: the role of larval competition. Ecology 78: 1.736-1.751.

LAVÍN-MURCIO, P.; DIXON, J.R. 2004. A new species of coral snake (Serpentes: Elapidae) from the Sierra de Tamaulipas, Mexico. Phyllomedusa 3:3-7

LLANO-MEJÍA, J.; CORTÉS-GÓMEZ, A.M. ; CASTRO-HERRERA, F. 2010. Lista de anfibios y reptiles del departamento del Tolima, Colombia. Biota colombiana 11 (1 y 2): 89-106.

LYNCH, J.D. 2012. El contexto de las serpientes de Colombia con un análisis de las amenazas en contra de su conservación. rev. Colomb. Cienc. 36 (140): 435-4498.

LIPS, K.R.; REEVE, J.; WITTERS, L. 2003. Ecological factors predicting amphibian population declines in Central America. Conservation Biology 17:1078-1088.

MOSQUERA N.Y. 2016. Diversidad de ofidios presentes en tres zonas en el departamento del Chocó- Colombia. Trabajo de grado para optar el título de biólogo con énfasis en recursos naturales. UTCH. Biología. 
Pitalua-L et al - Aportes a la distribución del género Micrurus (Serpentes: Elapidae) Chocó, Colombia.

MURILLO M.F. 2006. Contribución al conocimiento de la Ofidiofauna de cuatro comunidades de la cuenca hidrográfica del río Cabí. Trabajo de grado para optar el título de Biólogo con énfasis en recursos naturales. UTCH. Biología.

PAEZ, U.P.; BRIAN, C.B. 2002. Guía de campo de algunas especies de anfibios y reptiles de Antioquia. EPM. Medellín, Colombia.

PALACIOS RODRIGUEZ, P.; RENGIFO, J.T 2012. Nueva localidad para Micrurus multiscustatus (serpiente: Elapidae) en la región natural del Chocó, Colombia. 31 (2): 96-99.

PÉREZ-SANTOS, C.; MORENO, A.G. 1988. Ofidios de Colombia. Museo Regionale di Scienze Naturali, Monographie VI. Torino, Italy.

PETERS, J.A.; OREJAS-MIRANDA, B. 1970. Catalogue of Neotropical Squamata. Part I. Snakes. Smithsonian Institution Bull. 297. Washington.

PHILLIPS, S.J.; ANDERSON, R.P.; SCHAPIRE, R.E. 2006. Maximum entropy modeling of species geographic distributions. Ecological Modelling 190 (3-4): 231-259.

PINCHEIRA-DONOSO, D.; BAUER, A.M.; MEIRI, S.; UETZ, P. 2013. Global taxonomic diversity of living reptiles. PLoS One 8: e59741.

POUNDS, J.A.; CRUMP, M.L. 1994. Amphibian declines and climate disturbance; the case of the golden toad and the harlequin frog. Conservation Biology 8:72-85.

POUNDS, J.A.; FOGDEN, M.P.; CAMPBELL, J.H. 1999. Biological response to climate change on a tropical mountain. Nature 398: 611-615.

POUNDS, J.A. PUSCHENDORF, R. 2004. Clouded futures. Nature 427:107-109.

PYRON, R.A.; BURBRINK, F.T.; WIENS, J.J. 2013. A phylogeny and revised classification of Squamata, including 4161 species of lizards and snakes. BMC Evolutionary Biology 13: $93-171$.

POVEDA M.C.; ROJAS. C.A.; RUEDAS. A.; RANGEL. J.O. 2004. El Chocó Biogeográfico: Ambiente físico. Págs. 1-22 En: Rangel. J.O (ed.). Colombia Diversidad Biótica IV: El Chocó Biogeográfico/Costa Pacífica. Universidad Nacional de Colombia. Bogotá. DC

RELYEA, R. 2005. The impact of insecticides and herbicides on biodiversity and productivity of aquatic communities. Ecological society of America: 618-627.

RENGIFO, T.; ASPRILLA, J.; RENGIFO, A.; JIMÉNEZ ROA, Y. 2004. Hacia una aproximación a la herpetofauna (Anfibios y Reptiles) del municipio de Novita, departamento del Chocó, Colombia. Revista Institucional Universidad Tecnológica del Chocó 20: 39-44.

RENGIFO F, ASPRILLA, J.; JIMÉNEZ, A.J.; RENGIFO, J.; CASTRO, A. 2002. Ecología y estructura de la comunidad de reptiles presentes en el corregimiento de Pacurita, municipio de Quibdó, Chocó, Colombia. Seminario Internacional del Medio Ambiente y Desarrollo Sostenible, 2002. División Editorial y Publicaciones, Universidad Industrial de Santander. Bucaramanga, Colombia.

RENGIFO, M.J.T.; RENTERIA, M.L.E. 2011. Reptiles del departamento del Chocó, Colombia. rev. Biodivers. Neotrop. 1 (1): 38-47. 
RIBEIRO-JÚNIOR, M.A.; GARDNER, T.A.; AVILA-PIRES, T.C.S. 2008. Evaluating the effectiveness of herpetofaunal sampling techniques across a gradient of habitat change in a tropical forest landscape. Journal of Herpetology 42: 733-749.

ROZE, A.J. 1996. Coral snake of the Americas. Biology, identification, and venoms. American Museum of Natural History. Krieger Publishing Company. Malabar, Florida.

RUEDA-ALMONACID, J.V.; RUEDA-MARTÍNEZ J.N. 2004. Primer registro de una serpiente del género Amastridium Cope (Serpentes: Colubridae), para Sudamérica. rev acad Colomb Cienc. 28 (107): 291-3.

SAVAGE J.M. 2002. The amphibians and reptiles of Costa Rica: A herpetofauna between two continents, between two seas. Chicago: University of Chicago Press. 934 pp. Review in Copeia 2003 (1): 205.

STUART, S.N.; CHANSON, J.S.; COX, N.A.; YOUNG, B.E.; RODRÍGUEZ, A.S.L.; FISCHMAN, D.L.; WALLER, R.W. 2004. Status and trends of amphibian declines and extinctions worldwide. Science 306: 1783-1786.

UETZ, P.; HALLERMANN, J. 2014. The jcvi/tigr reptile database. Disponible en: http://reptiledatabase.reptarium.cz. Consultado: 25-10-2017.

UETZ, P.; FREED, P.; JIRÍ, H. 2016. The reptile database. Disponible en: http://www.reptiledatabase.org. Consultado: 25-10-2017.

VIDAL. N.; HEDGES, S.B. 2002. Higher-level relationships of snakes inferred from four nuclear and mitocondrial genes. C.R. Biologies 325: 977-985.

YOUNG, B.; LIPS, K.; REASER, J.; IBAÑES, R.; SALAS, A.; CEDEÑO, J.; COLOMA, L.; RON, J.; LA MARCA, E.; MUÑOS, A.; BOLAÑ̃S, F.; CHAVES, G.; ROMO, D. 2001. Population declines and priorities for amphibian conservation in Latin America. Conservation Biology 21(1): 95-109

YOUNG, B.E.; STUART, S.N.; CHANSON, J.S.; COX, N.A.; BOUCHER, T.M. 2004. Disappearing jewels: the status of new world amphibians. Nature Serve. Arlington, Virginia.

ZAHER, H. ; GRAZZIOTIN, F.G.; CADLE, J.E. ; MURPHY, R.W. ; MOURA-LEITE, J.C.; BONATTO, S.L. 2009. Molecular phylogeny of advanced snakes (Serpentes, Caenophidia) with an emphasis on South American Xenodontines: a revised classification and descriptions of new taxa. Papéis Avulsos de Zoologia. Museu de Zoologia da Universidade de São Paulo 49:115-153. 